\title{
Passive High-speed Imaging of Ion Acoustic Turbulence in a Hollow Cathode
}

\author{
Marcel P. Georgin*, Matthew P. Byrne † Nikolas E. Ciaston $\ddagger$ Benjamin A. Jorns \\ Alec D. Gallimore \\ University of Michigan, Ann Arbor, MI, 48109, USA
}

\begin{abstract}
A passive, non-invasive plasma diagnostic was developed and validated to estimate the dispersion relation of propagating electrostatic modes in a hollow cathode discharge. This new technique used a Silicon photomultiplier (SiPM) array to measure fluctuations in light intensity and relate them to fluctuations in plasma density. The development and validation of the SiPM technique is discussed in the context of non-invasive measurements of plasma waves. This technique is compared to a similar method using ion saturation probes to measure density fluctuations. Ion acoustic waves, believed to be responsible for the anomalous resistivity of the cathode plume, were measured using both ion saturation probes and the SiPM technique. While the raw data and power spectra acquired with the SiPM were different from the probes, the estimated dispersion relations were similar and the phase velocities, were within uncertainty of each other. These measurements were compared to similar measurements found in the literature to confirm that ion acoustic turbulence was measured. The weaker signal to noise in the SiPM signal is shown to most likely be a result of spatial averaging. The results suggest that the SiPM technique may be capable of estimating the dispersion of high-frequency waves in other plasma devices.
\end{abstract}

\section{Nomenclature}

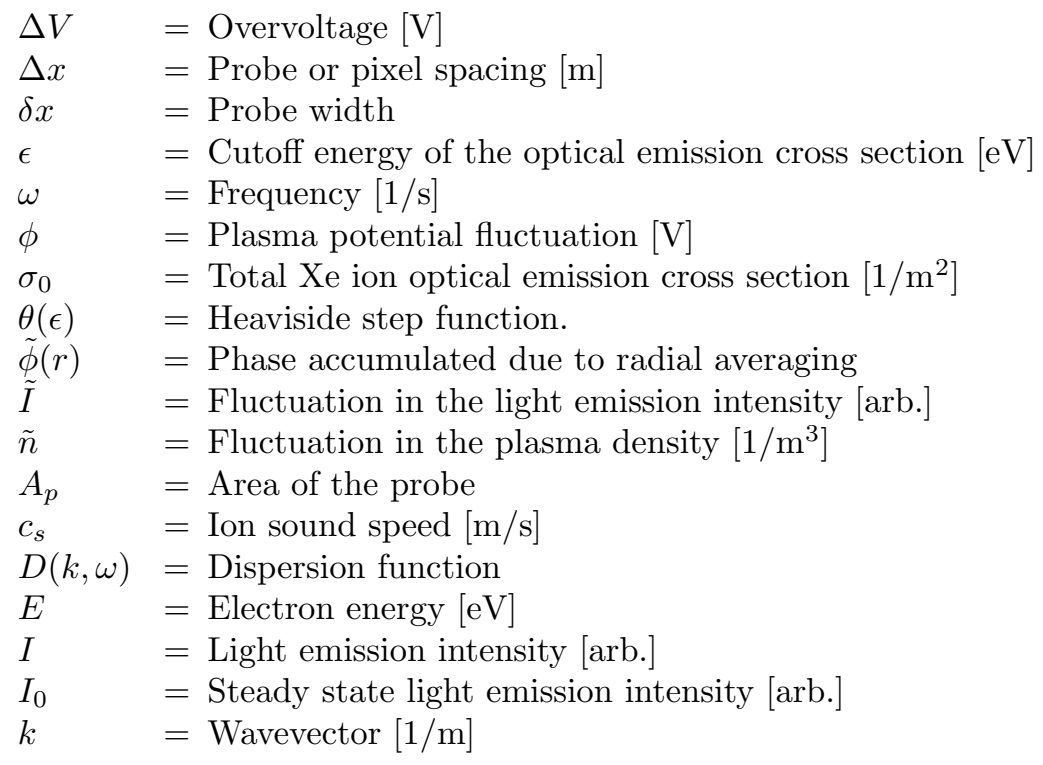

${ }^{*}$ Ph.D. Candidate, Applied Physics, AIAA Student Member.

$\dagger$ Ph.D. Pre-Candidate, Applied Physics, AIAA Student Member.

$\ddagger$ Master’s Student, Aerospace Engineering, AIAA Student Member.

$\S$ Assistant Professor, Aerospace Engineering, AIAA Senior Member.

I Robert J. Vlasic Dean of Engineering, the Richard F. and Eleanor A. Towner Professor of Engineering, and an Arthur F. Thurnau Professor of Aerospace Engineering, and AIAA Fellow. 


$\begin{array}{ll}M & =\text { Number of trials } \\ m_{i} & =\text { Ion mass }[\mathrm{kg}] \\ n & =\text { Plasma density }\left[1 / \mathrm{m}^{3}\right] \\ n_{0} & =\text { Steady state plasma density }\left[1 / \mathrm{m}^{3}\right] \\ n_{n} & =\text { Neutral density }\left[1 / \mathrm{m}^{3}\right] \\ P(\omega) & =\text { Power spectrum }[\mathrm{arb} .] \\ q & =\text { Electron charge }[\mathrm{C}] \\ T_{e} & =\text { Electron temperature }[\mathrm{eV}] \\ u_{i} & =\text { Ion drift velocity }[\mathrm{m} / \mathrm{s}] \\ v_{p h} & =\text { Phase velocity }[\mathrm{m} / \mathrm{s}]\end{array}$

\section{Introduction}

P LASma instabilities have been studied in electric propulsion devices for the past several decades. ${ }^{1,2}$ These P waves can have characteristic frequencies from $1 \mathrm{kHz}$ to above $10 \mathrm{MHz} .{ }^{1}$ Some oscillations, such as the breathing mode in Hall thrusters, can be sufficiently large that the device cannot sustain its discharge. Other types of instabilities play a role in the fundamental operation of these devices. For example, ion acoustic waves have been measured in hollow cathodes and are believed to anomalously increase the resistivity of the plasma. ${ }^{3}$ Waves similar to ion acoustic waves have also been measured in the near-field plume of a Hall thruster and are thought to contribute to the cross-field transport of electrons in that region of the discharge. ${ }^{4}$ The ability to measure the properties of plasma instabilities can inform our understanding of the fundamental operation of electric propulsion devices.

To date, a few plasma diagnostics have been used to measure wave properties in Hall thrusters. First, electrostatic probe arrays that collect ion saturation current were used to characterize longitudinal and azimuthal modes in the discharge channel. ${ }^{5-7}$ Although probes can directly measure relative plasma density fluctuations, placing them in the channel of a Hall thruster is known to perturb the operation of the thruster and sometimes even terminate the discharge. ${ }^{8,9}$ These perturbative effects limit where probes can be used and motivate the development of non-invasive measurements of instabilities. High-speed CCD cameras have been used to image fluctuations in the light emission from azimuthal modes in Hall thrusters. ${ }^{10,11}$ This technique has limited spatial resolution, because it measures the integrated light emission from the plasma. Furthermore, the bandwidth of high-speed cameras is typically $<1 \mathrm{MHz}$, making this technique only suitable for measurements of low frequency oscillations. The low bandwidth of this technique means that it cannot be used to measure the high frequency oscillations that are believed to influence electron transport in Hall thrusters. Coherent Thomson scattering is the only non-invasive technique used to make measurements of wave properties in a Hall thruster channel to date. ${ }^{4}$ While the technique has produced the first evidence of ion-acoustic-like modes in the channel, it is limited in the wavelengths it can measure and requires spatial integration to recover a signal. The setup required for this diagnostic is also expensive and difficult to build. Although the available techniques are able to make measurements of high frequency modes, there is an apparent need for an inexpensive and non-invasive plasma diagnostic that has sufficient bandwidth and wavelength resolution to measure these high-frequency instabilities. This work develops and validates a new technique to measure high frequency fluctuations in emitted light intensity.

The new method for measuring fluctuations in light emission is similar to the technique described by Pots. ${ }^{12}$ Pots measured light fluctuations by collecting the emission from the plasma, diffracting it through a grating, and sending it through fiber optics to an array of photomultiplier tubes (PMTs) for measurement. Our technique uses a silicon photomultiplier ( $\mathrm{SiPM}$ ) array and lenses to image the plasma. An SiPM is a semiconductor chip that can be used in a similar way to a traditional photomultiplier to detect light. These SiPMs have a large gain $\left(10^{6}\right)$ and can be purchased in an array of up to $8 \times 8$. The spatial distribution of the SiPM array allows several points to be measured simultaneously at frequencies up to $4 \mathrm{MHz}$.

Ion saturation probes have been used to successfully estimate the dispersion relation of ion acoustic turbulence (IAT) in the plume of a hollow cathode. ${ }^{3}$ The probes rely on an analysis technique developed by Beall to estimate the dispersion relation. ${ }^{13}$ This method requires the simultaneous measurement of two points in the plasma to compute the wavevector, $k$ at a frequency $\omega$. This previous measurement of IAT makes the cathode plume a convenient testbed for our new non-invasive technique for estimating the dispersion relation. IAT manifests itself at frequencies between $100 \mathrm{kHz}$ and $5 \mathrm{MHz}$. The SiPM should have sufficient bandwidth to cover that frequency range. By imaging the plasma on two SiPM pixels, the dispersion relation 
of the plasma may be estimated using the same analysis technique as the probes. The results from the ion saturation probes and SiPM can be directly compared.

First, we will discuss the operating principles of both the ion saturation probe and SiPM techniques and the expectation for the dispersion. Next, the experimental setup and the calibration of the SiPM diagnostic are described. Lastly, the SiPM technique is bench-marked against the ion saturation probe method by measuring IAT in a hollow cathode. This comparison explores the fundamental limitations and potential future applications of this diagnostic for non-invasive plasma measurements in electric propulsion devices.

\section{Principle of Operation}

The fundamental principles of operation of the ion saturation probes and the SiPM are discussed in this section. An analysis of the light emission shows, under some simplifying assumptions, that the SiPM technique can be analyzed in the same way as the ion saturation probes. Lastly, the estimation of the dispersion relation using the Beall technique is discussed.

\section{A. Ion Saturation Probes}

Ion saturation probes measure the ion saturation current by negatively biasing an electrode in a plasma. Jorns et al. experimentally confirmed the presence of ion-acoustic turbulence using a pair of ion saturation probes. ${ }^{3}$ Under the assumption of a low-frequency and low-amplitude oscillation, the fluctuation of the plasma potential for an electrostatic mode, such as ion-acoustic waves, can be related to plasma density fluctuations by

$$
\phi \simeq \frac{T_{e}}{q} \frac{\tilde{n}}{n_{0}},{ }^{14}
$$

where $T_{e}$ is the electron temperature, $\tilde{n}$ is the fluctuation in the plasma density and $n_{0}$ is the equilibrium plasma density. The ion saturation current collected by a probe is given by

$$
i_{\text {sat }}=0.61 q A_{p} \sqrt{\frac{T_{e}}{m_{i}}} n,
$$

where $A_{p}$ is the surface area of the probe. Since the ion saturation current is proportional to the plasma density, fluctuations in the ion saturation current can be used to estimate the fluctuation in the plasma potential, assuming the oscillation in the electron temperature is small

$$
\phi \simeq \frac{T_{e}}{q} \frac{\tilde{i}_{\text {sat }}}{\bar{i}_{\text {sat }}},
$$

where the $\tilde{i}_{\text {sat }}$ is the fluctuation in the ion saturation current and $\bar{i}_{\text {sat }}$ is the steady state ion saturation current. By computing the correlation between the signals from two spatially separated saturation probes, the dispersion, $D(k, \omega)$, of the wave can be estimated using Beall analysis. ${ }^{13}$

\section{B. Silicon Photomultiplier}

In principle, a similar technique could be developed by measuring fluctuations in light intensity. Below is an analysis that shows the equivalence of measuring fluctuations in light intensity and ion saturation current under certain assumptions. As a proof of concept, an SiPM array was used to passively and non-invasively measure the ion-acoustic instability present in high current hollow cathode plumes ${ }^{3,15}$. The goal is to measure high frequency fluctuations in the light intensity to estimate wave properties such as the dispersion and phase velocity. The intensity of the light, $I$ produced by the cathode should be ${ }^{16}$

$$
I=n_{n} n \int_{0}^{\infty} \sqrt{\frac{2 q E}{m_{e}}} \sigma_{e m i s}(E) f(E) d E
$$

where $E$ is the electron energy and $\sigma_{\text {emis }}$ is the optical emission cross-section. For simplicity, if we assume that the plasma is Maxwellian then the expression simplifies to

$$
I \simeq n_{n} n T_{e}^{-3 / 2} \int_{0}^{\infty} \sigma_{e m i s}(E) e^{-\frac{E}{T_{e}}} E d E .
$$


The optical emission cross-section for xenon vanishes below a threshold energy $\epsilon$, which is about $10 \mathrm{eV}^{17}$. Since the electron temperature in the cathode plume is about $3 \mathrm{eV}$, only the high energy tail of the distribution will generate optical excitation. Approximating the $\sigma_{e m i s} \simeq \sigma_{0} \theta(\epsilon)$ and computing the integral we find

$$
I \simeq n_{n} n \sigma_{0} \sqrt{T_{e}} e^{-\frac{\epsilon}{T_{e}}}\left(1+\frac{\epsilon}{T_{e}}\right)
$$

If we assume that the electron temperature is on the same order as the cut-off energy, then we can series expand the exponential as

$$
I \simeq n_{n} n \sigma_{0} \sqrt{T_{e}}\left(1+\frac{T_{e}}{\epsilon}\right)
$$

Equation 7 shows that the optical emission of the plasma is proportional to the neutral and plasma densities and the electron temperature. Fluctuations in any of these quantities should result in changes in the intensity of optical emission. The neutral density can only fluctuate due to the changes in the ionization rate, which is much smaller than the characteristic frequencies of the ion-acoustic modes. ${ }^{1,3}$ Assuming that the neutral density and the electron temperature are not fluctuating (the same assumption as the probe technique), then the relative fluctuation in optical emission is proportional to the relative fluctuation in plasma density:

$$
\frac{\tilde{I}}{I_{0}} \simeq \frac{\tilde{n}}{n_{0}}
$$

where $I_{0}$ and $n_{0}$ are the steady state light intensity and plasma density and $\tilde{I}$ and $\tilde{n}$ are the fluctuations in light intensity and plasma density. Equation 8 allows us to exchange the ion saturation current measurement from the probes with the light intensity fluctuation measurement from the SiPM detector and as the proxy for density fluctuations,

$$
\phi \simeq \frac{T_{e}}{q} \frac{\tilde{I}}{I_{0}} .
$$

By similarly computing the correlation between the two pixels, a map of the dispersion relation can be generated using the technique developed by Beall ${ }^{13}$. This analysis makes similar assumptions to those for the ion saturation probes; however, the caveat is that the measured intensity is both line integrated through the plasma and spatially averaged over the image of the plasma in the pixel.

This new experimental technique is validated against measurements from the ion saturation probe method and is used to estimate wave properties in a 20 A hollow cathode designed at the Plasmadynamics and Electric Propulsion Laboratory (PEPL). ${ }^{18}$ This investigation examines both the validity of the new diagnostic and searches for ion-acoustic waves in hollow cathodes at lower currents 45-55 A compared to the measurements made by Jorns et al. with a 100-A hollow cathode. ${ }^{3}$

\section{Analysis Technique}

The measured traces from the probes and the SiPM are analyzed using a dispersion estimation technique similar to that developed by Beall ${ }^{13}$. The technique is predicated on the eikonal approximation: ${ }^{3}$

$$
\phi=\sum_{\omega} \phi(\omega) e^{i(k(\omega) x-\omega t)}+\text { c.c. },
$$

which assumes that for frequency $\omega$, there exists one wavevector $k(\omega)$. By capturing a signal from two probes (or SiPM pixels) with a fixed spacing, $x_{1}-x_{2}=\Delta x$, the signals can be decomposed using Eqn. 10 as

$$
\begin{aligned}
& \phi_{1}=\sum_{\omega} \phi_{1}(\omega) e^{i\left(k(\omega) x_{1}-\omega t\right)}+c . c . \\
& \phi_{2}=\sum_{\omega} \phi_{2}(\omega) e^{i\left(k(\omega) x_{2}-\omega t\right)}+c . c .
\end{aligned}
$$

This technique estimates the wavevector in the direction of the probe separation by finding the phase between two signals at a known probe separation. In a plasma, however, there can be multiple modes present and the 
modes have random phase with limited coherence. Therefore a single estimation of the wavenumber for a particular frequency may be errant. To achieve a more realistic estimation of the dispersion in a plasma, we will employ the statistical analysis first proposed by Beall to determine a two dimensional power spectrum in $\omega$ and $k$ space. The power spectrum in $\omega$ space, $P(\omega)$, is found by computing the Fourier transform of the time trace. To produce a power spectrum in $k$ space, $P(k), k(\omega)$ must be estimated $M$ times and binned. To produce the two dimensional power spectrum, $S(\omega, k), P(k)$ must be weighted by the power spectrum $P(\omega)$. Because $k(\omega)$ is estimated from two traces, $P(\omega)$ should the average power spectrum of the traces. Mathematically the binning is written as

$$
S(k, \omega)=\frac{1}{M} \sum_{j=1}^{M} I_{0, \Delta k}\left(k-k^{j}(\omega)\right) \frac{1}{2}\left(P_{1}^{j}(\omega)+P_{2}^{j}(\omega)\right),
$$

where $j$ indicates a run number and the function

$$
I_{0, \Delta k}\left(k-k^{j}(\omega)\right)=\left\{\begin{array}{ll}
1 & \left|k-k^{j}(\omega)\right|<\Delta k \\
0 & \left|k-k^{j}(\omega)\right|>\Delta k
\end{array},\right.
$$

where $\Delta k$ is the bin width. Using this statistical description to estimate the dispersion relaxes the original assumption that there exists a single $k(\omega)$ and allows us to visualize the dominant dispersion content of the plasma.

Based on this analysis technique, ${ }^{3}$ the maximum $k$ that can be observed by the probes (or SiPM) is related to the distance between the two sampling points:

$$
k_{\max }=\min \left\{\begin{array}{l}
\frac{\pi}{\Delta x} \\
\frac{2 \pi}{\delta x}
\end{array},\right.
$$

where $\Delta x$ is the spacing between the probes and $\delta x$ is the probe width. These conditions are similar to the Nyquist frequency when measuring a time dependent signal. The technique generates the dispersion relation in modulo $k_{\max }$, therefore measurements that exceed this maximum observable value of $k$, are aliased or "phase wrapped" to negative $k$. To correct for this, the dispersion relation can be concatenated to extend the domain of $k$.

\section{Ion-Acoustic Turbulence}

In this work, we will be looking for ion-acoustic waves in the laboratory frame and in the most unstable direction. The real component of the frequency is given by

$$
\omega_{r}=\left(c_{s}+u_{i}\right) k,
$$

where $c_{s}$ and $u_{i}$ are the ion sound speed and the ion drift velocity. ${ }^{15,19}$ The ion sound speed is given by

$$
c_{s}=\sqrt{\frac{2 q T_{e}}{m_{i}}} .
$$

Previous measurements in hollow cathodes suggest that the phase velocity, $\omega / k$ of the wave should be approximately $6000 \mathrm{~m} / \mathrm{s}^{3}$

\section{Experimental Methods}

The following is a description of the experimental configuration for the hollow cathode experiment and details about the measurement scheme for both the probes and the SiPM array.

\section{A. Experimental Configuration}

The hollow cathode used for this experiment is the $\mathrm{LaB}_{6}$ hollow cathode designed at PEPL. The $\mathrm{LaB}_{6}$ insert has a $3 \mathrm{~cm}^{2}$ surface area and is held within a graphite cathode tube. This cathode uses a serpentine heater that is electrically isolated by boron nitride spacers. The orifice plate is made of tungsten and the keeper 
is made of graphite. The plasma discharge connects to a water-cooled tungsten anode $3.8 \mathrm{~cm}$ away that was provided by the electric propulsion group at NASA's Glenn Research Center. The cathode's nominal operating current is $20 \mathrm{~A}$, however in this work the cathode was operated between 45 and $55 \mathrm{~A}$ at $5 \mathrm{sccm}$ to induce the IAT.

The cathode was tested in the cathode test facility (CTF) at PEPL. The facility is $40 \mathrm{~cm}$ in diameter, $1 \mathrm{~m}$ long and uses a cryogenic pump with a pumping speed of $1500 \mathrm{~L} / \mathrm{s}$ on xenon to achieve a base pressure of about $1 \times 10^{-6}$ Torr- $\mathrm{N}_{2}$. The typical operating pressures during cathode testing was $2 \times 10^{-4}$ Torr-Xe.

\section{B. Ion Saturation Probe Array}

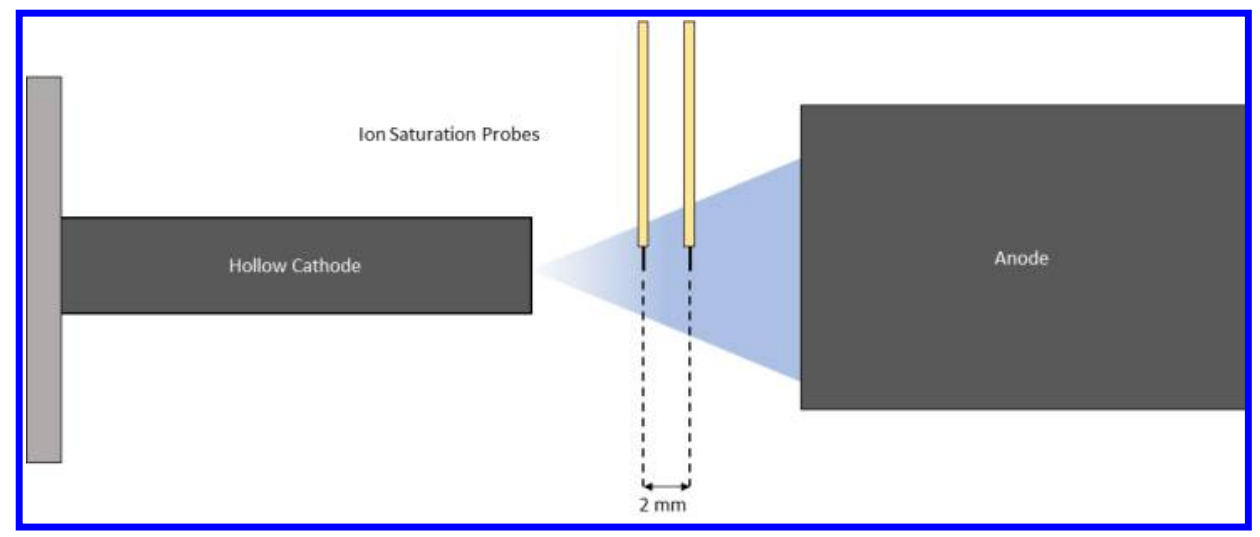

Figure 1: Ion saturation probe setup. The probes are spaced $2 \mathrm{~mm}$ apart and $2 \mathrm{~cm}$ from the cathode.

The saturation probe array is shown in Fig. 1. Probe 1 is nearest to the anode and probe 2 is $2 \mathrm{~mm}$ upstream. These probes were used to estimate the dispersion of the plasma in the axial direction. The tungsten electrode is $0.5 \mathrm{~mm}$ in diameter and protrudes $2 \mathrm{~mm}$ from the ceramic. Based on these dimensions and Eqn. $15, k_{\max }=15701 / \mathrm{m}$. The probes were placed at $2 \mathrm{~cm}$ from the cathode. An electrical schematic is shown in Fig. 2. The bias voltage to the probes was $-36 \mathrm{~V}$ using batteries in series and the saturation current measurements were made using a commercially available oscilloscope across a $100 \Omega$ resistor. During this experiment, the probes were fixed in position.

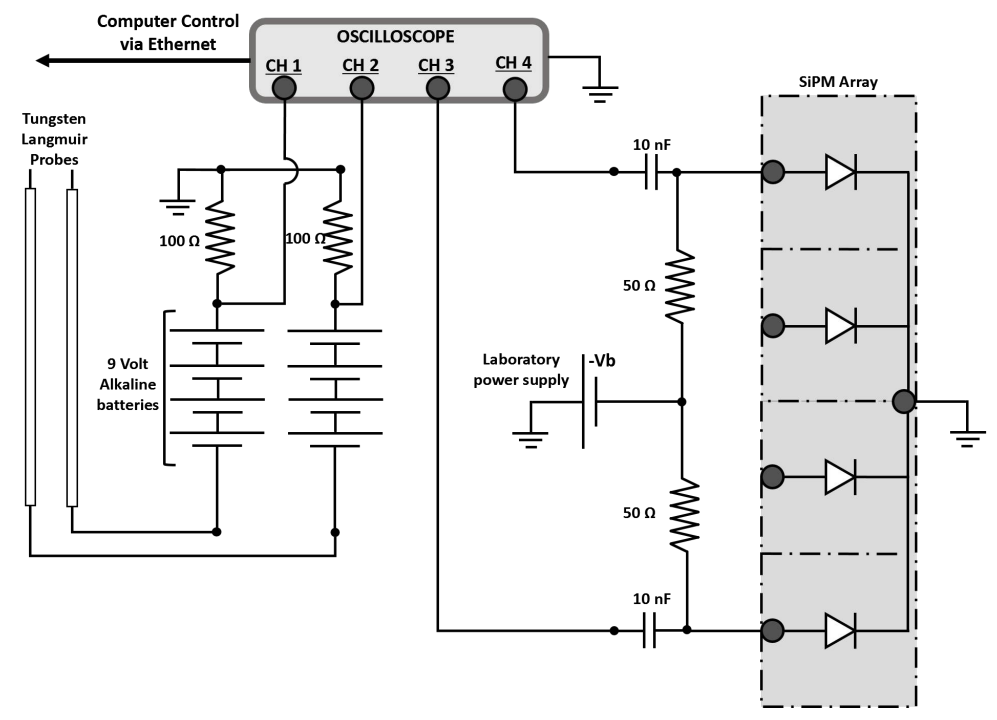

Figure 2: Circuit diagram of the experimental setup showing both the probes and SiPM. 


\section{Silicon Photomultiplier Array}

The SensL inc. ArrayJ-30035-16P-PCB SiPM array, used in this study, is a $4 \times 4$ array of 16 J-series SiPMs, which are $3 \times 3 \mathrm{~mm}^{2}$ pixels. Each SiPM in the array is the functional equivalent to a photomultiplier tube (PMT), capable of achieving a gain greater than $10^{6}$. The gain is controlled by the overvoltage, the amount of reverse voltage applied above the diode breakdown voltage. The gain is proportional to the overvoltage, with overvoltages of 2.5 to $5 \mathrm{~V}$ leading to typical gains of between $2.8 \times 10^{6}-5.3 \times 10^{6} .{ }^{20}$ For a given overvoltage, the sensor will eventually saturate with increased light intensity and the response will be non-linear. The linear region of the SiPM is expected to be below $70 \%$ of the maximum output current. ${ }^{21}$ For the $3 \times 3 \mathrm{~mm}^{2}$ pixels used in this experiment, the maximum output current is about $20 \mathrm{~mA}$, therefore the saturation region should begin at around $14 \mathrm{~mA}$. The linearity of the SiPM output was measured using an LED and neutral density filters to reduce the light intensity. The results of this test are shown in Fig. 3a, which indicates that at higher intensities the response of the SiPM may be beginning to saturate. The J-series SiPM has

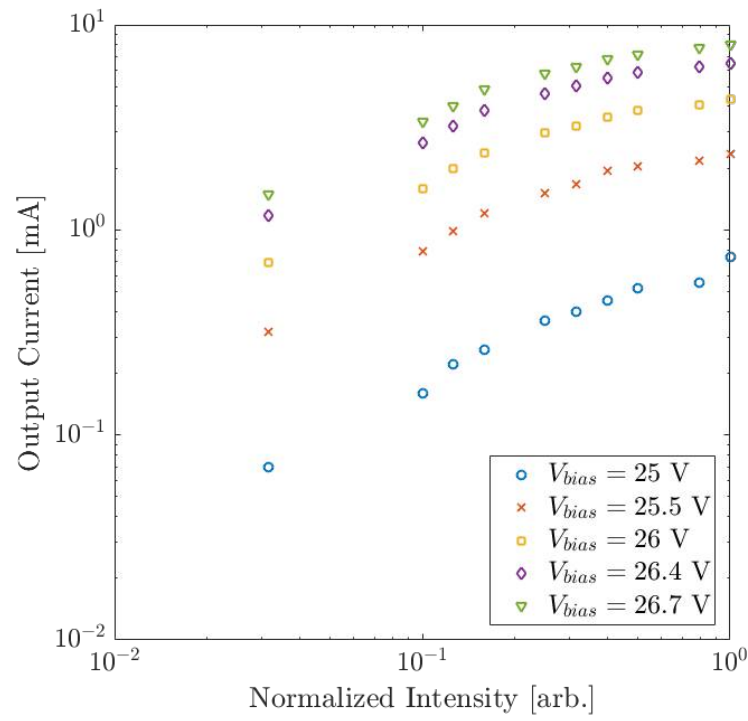

(a) SiPM response to varying light intensity at various bias voltages.

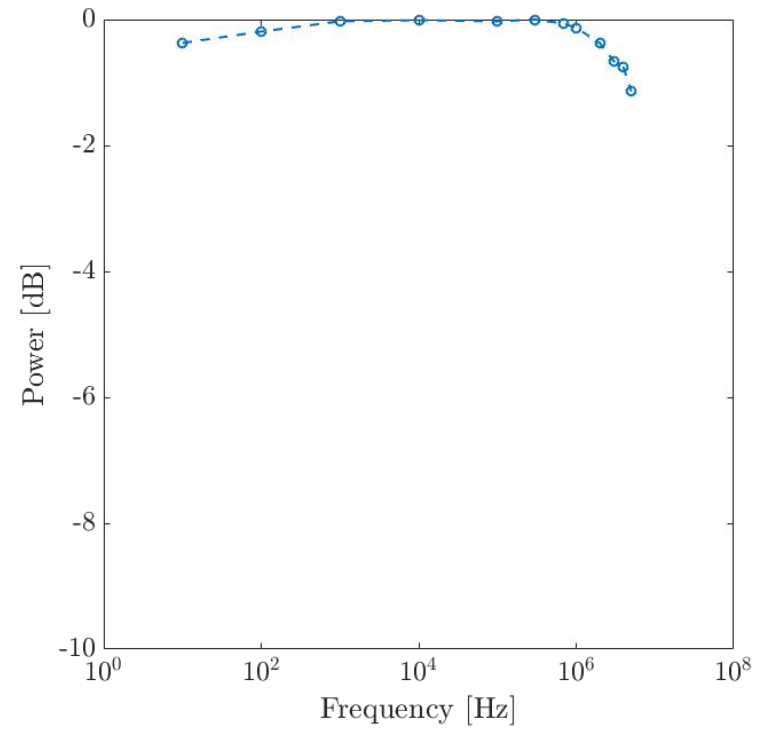

(b) Frequency response curve of the SiPM at LED driving frequencies between 1 and $5 \mathrm{MHz}$.

Figure 3: Intensity and frequency response curves for the SiPM setup.

spectral range of 200 to $900 \mathrm{~nm}$ with peak photon detection efficiency at $420 \mathrm{~nm} .{ }^{20}$ It also has a large frequency response, with a cutoff frequency of $4.3 \mathrm{MHz}$ in standard output mode. This frequency response is estimated by the published pulse width of a single microcell in a pixel. ${ }^{20}$ The frequency response of our SiPM setup was measured by oscillating an LED between $10 \mathrm{~Hz}$ and $5 \mathrm{MHz}$ and measuring the output of the SiPM. The frequency response curve is depicted in Fig. $3 \mathrm{~b}$ and shows that the frequency cut off $(-1 \mathrm{~dB})$ is at about $4 \mathrm{MHz}$, as expected from the pulse width. In addition to the upper frequency cutoff, there is also a lower frequency cutoff around $10 \mathrm{~Hz}$ due to the decoupling capacitor in the measurement scheme of the AC component.

The experimental configuration for the SiPM is shown in Fig. 4. The SiPM detector was placed outside of the CTF and imaged the cathode through an acrylic window. The light was focused onto the SiPM array using an achromatic doublet. The SiPM cells were biased with a laboratory power supply and the output current was measured through a decoupling capacitor. The voltage measured by the scope proportional to current produced by the SiPM. Figure 2 shows the electrical schematic for the SiPM and probe measurements. With this experimental setup, each pixel collects approximately a $1 \times 1 \mathrm{~mm}^{2}$ space in the plume of the cathode, or a magnification of 3 . The two pixels used for the dispersion analysis were $9 \mathrm{~mm}$ apart from their centers making the collected light $3 \mathrm{~mm}$ apart in the plume. The SiPM was focused upstream of the probe setup to avoid reflections. Based on Eqn. 15, $k_{\max }$ for this configuration is $10471 / \mathrm{m}$. 


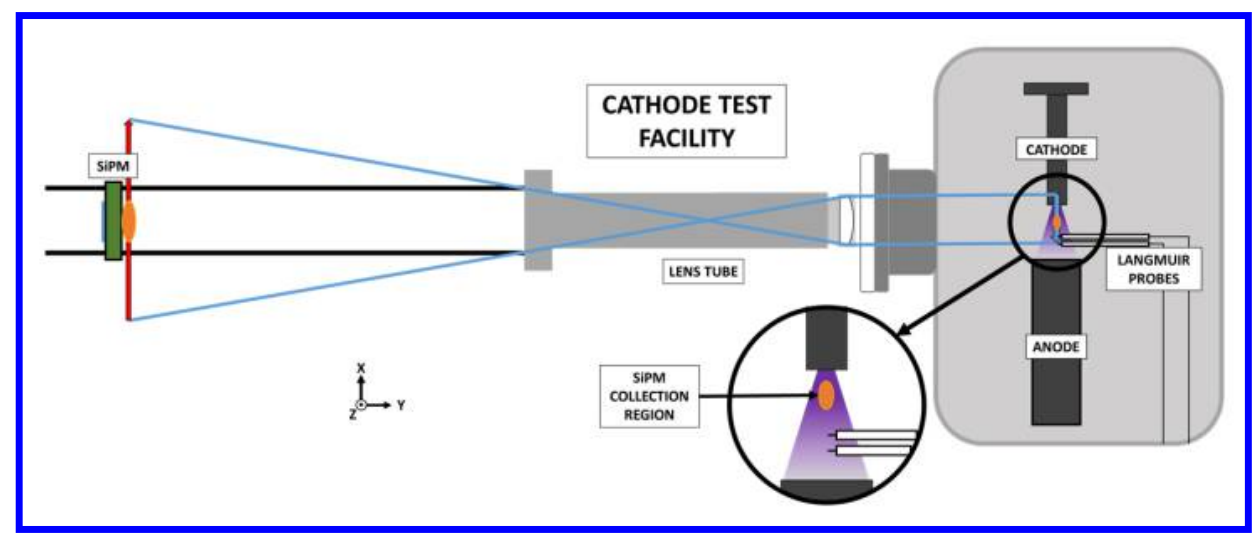

Figure 4: Silicon photomultiplier setup. The cathode plume is imaged onto the SiPM by an achromatic lens.

\section{Results \& Discussion}

The following discussion describes the differences between the probe and optical techniques. The figures below show results for the $55 \mathrm{~A}, 20 \mathrm{~V}, 5 \mathrm{sccm}$ test condition. First, we will show the the raw response of the probes and SiPM array. Raw measurements are shown in Fig. 5. Fig 5a shows the response from the probes. Qualitatively, the two probes have similar traces separated by a phase offset, as expected for a traveling wave. Fig. 5b shows the response from the two SiPM pixels. In the SiPM traces a 50-kHz oscillation appears. The higher frequency oscillations do not show a clear phase relationship between the two pixels. From the raw traces, there are already some apparent differences in the response to plasma oscillations.

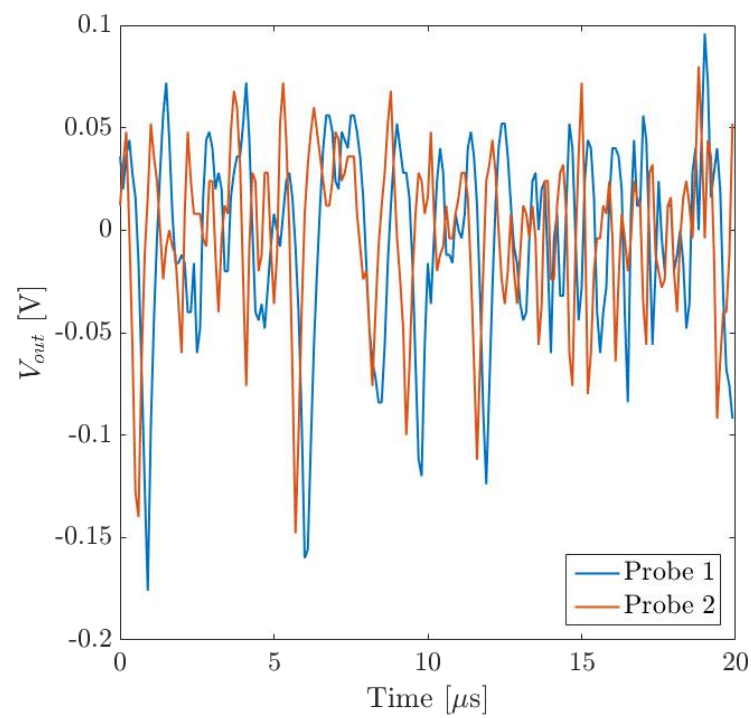

(a) Raw voltages measured by the probes.

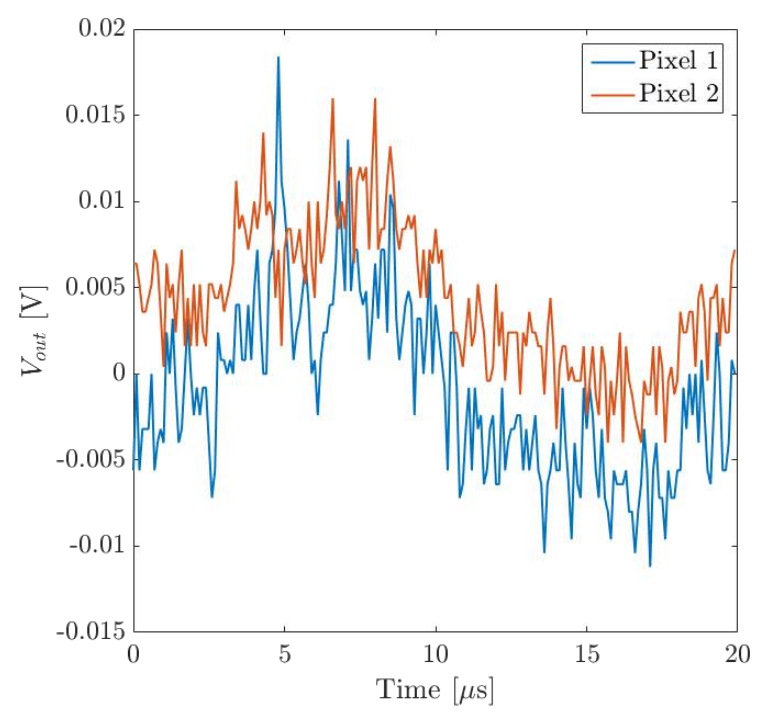

(b) Raw voltages measured by the SiPM.

Figure 5: Comparison of the raw probe and SiPM signal over the first $20 \mu$ s during cathode operation at 55 A and $5 \mathrm{sccm}$.

To quantify these discrepancies, we Fourier transforming the signals to explicitly examine the differences in the frequency content of the probes and the SiPM. The Fourier power spectrum is shown in Fig. 6 . The major differences between these two traces is the dominance of the $50-\mathrm{kHz}$ mode in the SiPM signal and the dominance of the broad oscillation in the probe signal near $1 \mathrm{MHz}$. This broad, high frequency content in the Fourier transform is associated with the presence of ion-acoustic turbulence. ${ }^{3}$ In the SiPM spectrum there exists a small concentration of power around $1 \mathrm{MHz}$ which may be a result of ion-acoustic wave detection. The damping of the IAT in the SiPM signal may be a result of line integration of the collected light through 
the plasma. The weak signal at high frequency in the SiPM will be further discussed later.

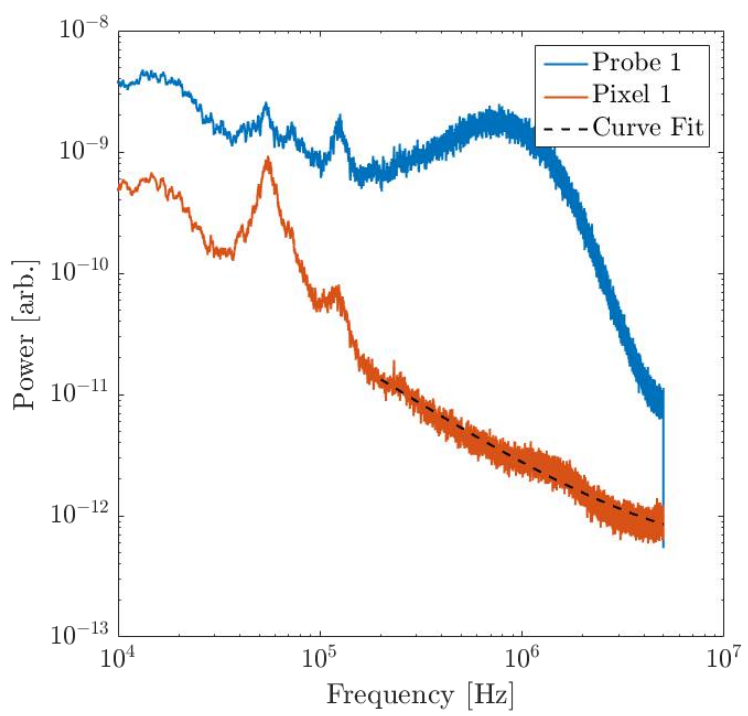

Figure 6: A comparison of the frequency content from probe 1 and pixel 1 on the SiPM at $55 \mathrm{~A}$ and $5 \mathrm{sccm}$. This shows a difference in the high frequency response. A power law fit is to the high frequency response. The curve fit is $y=5.36 \times 10^{-6} x^{-1.06}+4.23 \times 10^{-13}$.

Using the analysis technique presented in Sec. II C, the dispersion relation of the plasma was estimated using the probe and SiPM measurements. A comparison of the dispersion relation is shown in Fig. 7, where the subfigures are saturated to show detail and are corrected for phase wrapping. The colorbar is proportional to the relative intensity of a component of the wave. Fig. 7a shows an acoustic relationship between the wavevector and frequency, or $\omega \propto k$, for the probes. Fig. 7b shows that the dispersion estimated with the

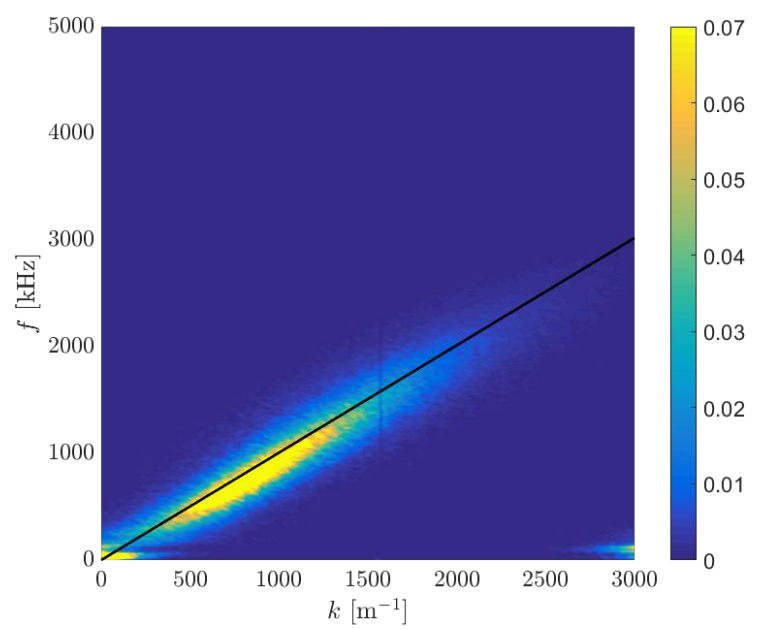

(a) Aliasing corrected data for the probes at the 55 A and $5 \mathrm{sccm}$ operating condition. The best fit line shows a phase velocity of $5.3 \pm 1.4 \mathrm{~km} / \mathrm{s}$.

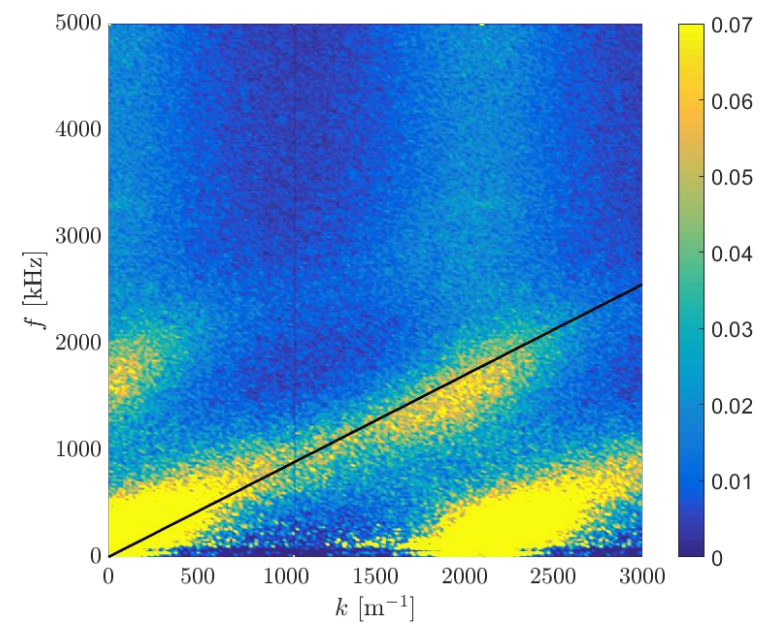

(b) Aliasing corrected data for the probes at the 55 A and $5 \mathrm{sccm}$ operating condition. The best fit line shows a phase velocity of $4.8 \pm 1.6 \mathrm{~km} / \mathrm{s}$.

Figure 7: Comparison of the phase wrapping corrected data between the probes and SiPM.

SiPM array is also acoustic in nature. The additional lobes seen in the SiPM signal are artifacts of the aliasing correction. The data was curve fit using Eqn. 16 to determine the phase velocity. For the $55 \mathrm{~A}$, $5 \mathrm{sccm}$ test condition shown in Fig. 7, the phase velocity was found to $5.3 \pm 1.4 \mathrm{~km} / \mathrm{s}$ for the probes and $4.8 \pm 1.6 \mathrm{~km} / \mathrm{s}$ for the SiPM. Although the dispersion relations and phase velocities estimated with each technique are similar, there are still some differences, most notably in the signal to noise ratio. The acoustic 
dispersion of the probe signal is more evident than the SiPM. Possible explanations for this are discussed in Sec. IV A.

The phase velocity found at a few current conditions is shown in Fig. 8. The uncertainty in the phase velocity is derived from the width of the probe (or SiPM). Below $50 \mathrm{~A}$, the IAT spectrum was not measurable with the SiPM. However, the measurements where IAT was observable show a similar trend to the probe measurements. Our data is compared to dispersion measurements made with probes on a 100 A cathode by Jorns et. al. ${ }^{3}$ Although these are measurements on two different cathodes the phase velocities are similar, suggesting that our results are the right order of magnitude.

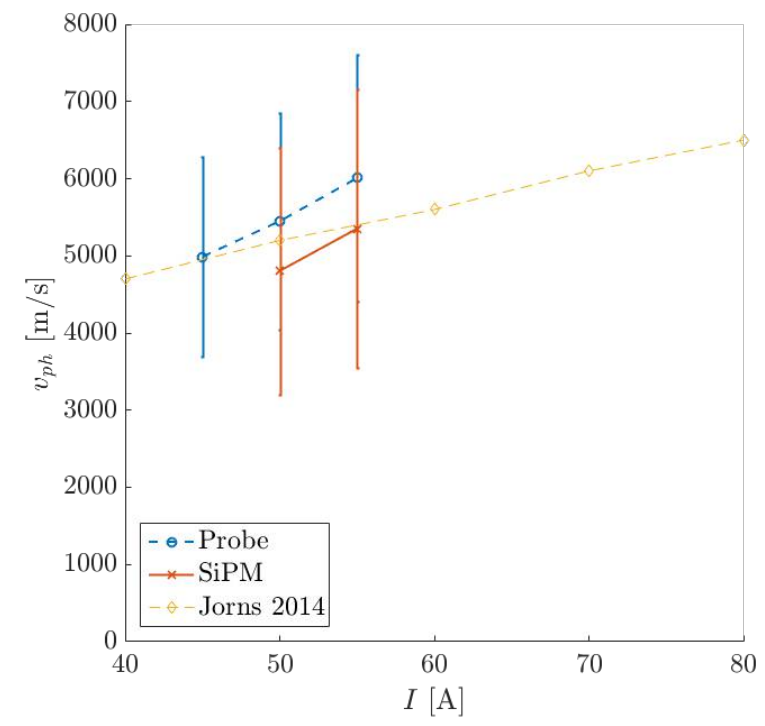

Figure 8: The measured phase velocity as a function of current at a mass flow rate of $5 \mathrm{sccm}$. Our results are the same order of magnitude as the reference data on a $100 \mathrm{~A}$ hollow cathode at $8 \mathrm{sccm}$. Probe data is in blue, SiPM in red and reference data is in yellow.

\section{A. Limitations of the SiPM Technique}

The dispersion relation measured by the SiPM is less apparent than the dispersion relation measured by the probes. Based on Fig. 3b, the sensitivity of SiPM at below $5 \mathrm{MHz}$ is at least $75 \%$ of the maximum. Therefore, the lack of signal is not due to a lack of sensitivity of the detector. There are a few possibilities that could explain the attenuation of the output signal of the SiPM, however the most likely cause is that the SiPM technique integrates the light intensity through the plasma.

Both the probe and SiPM technique rely on the assumption that the acoustic turbulence can be described as locally coherent, however the wave is globally incoherent. Because the SiPM method averages through the plasma, the incoherence of the IAT will damp the signal through destructive interference. To treat this mathematically, if we again use the eikonal approximation to describe the ion-acoustic waves and assume there some additional phase difference dependent on the radial position, we can write the wave as

$$
\phi=\sum_{\omega} \phi(\omega) e^{i(k(\omega) x+\tilde{\phi}(r)-\omega t)}+c . c .
$$

where $\tilde{\phi}(r)$ is the phase shift of the wave due radial position. Since the observed intensity by the SiPM is averaged over the diameter of the plasma, the amplitude measured at the SiPM is

$$
\phi_{S i P M}=\sum_{\omega} \phi(\omega) e^{i(k(\omega) x-\omega t)} \int_{0}^{R} \frac{e^{i \tilde{\phi}(r)} d r}{R}+c . c .,
$$

where $R$ is the radius of the plasma. If we assume that the phase $\tilde{\phi}(r)$ is proportional to the deviation off of the axis of the discharge, then

$$
\tilde{\phi}=k_{r} r
$$


where, $k_{r}$ is the radial component of the wavevector. After we integrate Eqn. 19 we find that the wave can be described by

$$
\phi_{S i P M}=\sum_{\omega} \frac{\phi(\omega)}{k_{r} R} \sin \left(k_{r} R\right) e^{i(k(\omega) x-\omega t)}+c . c .
$$

Since we are interested in acoustic modes, we would expect that $k_{r} \propto v_{p h} \omega$. Under these assumptions, we would expect that the amplitude of the Fourier spectrum should decay like

$$
\left|\phi_{S i P M}\right| \propto\left|\frac{1}{\omega}\right| .
$$

This treatment makes several strong assumptions, particularly the dependence of the phase on the radial position, but it shows that averaging through the plasma in the radial direction should lead to attenuation of the SiPM signal at higher frequencies.

Based on this analysis, the Fourier spectrum of the SiPM signal, shown in Fig. 6, was curve fit with a power law

$$
\phi_{S i P M}=m \omega^{-\alpha}+b .
$$

Only the section of the data where acoustic modes should have been present, $1 \mathrm{MHz}$ to $5 \mathrm{MHz}$, was used in the fit. The exponent, $\alpha$, was found to be $1.06 \pm 0.5$ which is in agreement with our simple model. The uncertainty in the exponent is derived from how much of the data is used in the fitting.

Overall, this analysis shows that spatial averaging reduces the amplitude of higher frequency waves. A potential way to improve this technique is to "tag" the ions using a laser, similar to laser induced fluorescence (LIF) experiments. This avenue will be explored further in future work on the development of this diagnostic.

\section{Conclusions}

We non-invasively estimated the dispersion relation in a hollow cathode discharge using an optical SiPM as a optical measurement device. Fluctuations in the light emitted from the plasma are shown to be proportional to the fluctuation in plasma density under the assumption of isothermality and small neutral density fluctuations. The dispersion measured with the SiPM technique is shown to be within uncertainty of the probe measurements. Futhermore, the phase velocity is shown to agree with reference data published by Jorns et al. ${ }^{3}$ The SiPM technique is advantageous over the probe technique in that it does not perturb the plasma, but the signal to noise suffers due to the phase averaging over line the integration and the is restricted by the image size on the SiPM pixels.

In addition, this new diagnostic can be used to implement a planar laser induced fluorescence (LIF) scheme to measure the spatial evolution of the ion velocity distribution. Further development may include a planar time-resolved LIF scheme that can resolve the Hall thruster breathing mode ${ }^{22}$. Lastly this diagnostic may permit the non-invasive estimation of dispersion in the near-field of a Hall thruster, which may provide clues to the electron cross field transport problem in Hall thruster numerical codes.

\section{Acknowledgments}

This work was funded by NASA Space Technology Research Fellowship grant NNX15AQ37H. The authors also would like to thank the electric propulsion group at NASA GRC for providing the anode for this experiment.

\section{References}

1 Choueiri, E. Y., "Plasma oscillations in Hall thrusters," Physics of Plasmas (1994-present), Vol. 8, No. 4, 2001, pp. 1411-1426.

$>^{2}$ Zhurin, V. V., Kaufman, H. R., and Robinson, R. S., "Physics of closed drift thrusters," Plasma Sources Science and Technology, Vol. 8, No. 1, 1999, pp. R1. 
$\triangleright^{3}$ Jorns, B. A., Mikellides, I. G., and Goebel, D. M., "Ion acoustic turbulence in a 100-A LaB6 hollow cathode," Physical Review E, Vol. 90, No. 6, Dec. 2014, pp. 063106.

-4 Tsikata, S., Lemoine, N., Pisarev, V., and Grsillon, D. M., "Dispersion relations of electron density fluctuations in a Hall thruster plasma, observed by collective light scattering," Physics of Plasmas, Vol. 16, No. 3, March 2009, pp. 033506.

${ }^{5}$ Chesta, E., Lam, C. M., Meezan, N. B., Schmidt, D. P., and Cappelli, M. A., "A characterization of plasma fluctuations within a Hall discharge," IEEE Transactions on Plasma Science, Vol. 29, No. 4, Aug. 2001, pp. 582-591.

6 Lazurenko, A., Coduti, G., Mazouffre, S., and Bonhomme, G., "Dispersion relation of high-frequency plasma oscillations in Hall thrusters," Physics of Plasmas (1994-present), Vol. 15, No. 3, March 2008, pp. 034502.

7 Litvak, A. A., Raitses, Y., and Fisch, N. J., "Experimental studies of high-frequency azimuthal waves in Hall thrusters," Physics of Plasmas (1994-present), Vol. 11, No. 4, April 2004, pp. 1701-1705.

8 Jorns, B., Goebel, D. M., and Hofer, R. R., "Plasma Perturbations in High-Speed Probing of Hall Thruster Discharge Chambers: Quantification and Mitigation," American Institute of Aeronautics and Astronautics, July 2015.

$\checkmark 9$ Grimaud, L., Petin, A., Vaudolon, J., and Mazouffre, S., "Perturbations induced by electrostatic probe in the discharge of Hall thrusters," Review of Scientific Instruments, Vol. 87, No. 4, April 2016, pp. 043506.

10 Jorns, B. A. and Hofer, R. R., "Plasma oscillations in a 6-kW magnetically shielded Hall thruster," Physics of Plasmas (1994-present), Vol. 21, No. 5, May 2014, pp. 053512.

-11 Sekerak, M. J., Longmier, B. W., Gallimore, A. D., Brown, D. L., Hofer, R. R., and Polk, J. E., "Azimuthal Spoke Propagation in Hall Effect Thrusters," IEEE Transactions on Plasma Science, Vol. 43, No. 1, Jan. 2015, pp. 72-85.

12 Pots, B. F. M., Turbulence and transport in a magnetized argon plasma, Eindhoven Tech. Univ., 1979.

$\checkmark 13$ Beall, J. M., Kim, Y. C., and Powers, E. J., "Estimation of wavenumber and frequency spectra using fixed probe pairs," Journal of Applied Physics, Vol. 53, No. 6, June 1982, pp. 3933-3940.

${ }^{14}$ Chen, F. F., "Langmuir probe diagnostics," IEEE-ICOPS Meeting, Jeju, Korea, Vol. 2, 2003.

-15 Jorns, B., Lopez Ortega, A., and Mikellides, I. G., "First-principles Modelling of the IAT-driven Anomalous Resistivity in Hollow Cathode Discharges I: Theory," American Institute of Aeronautics and Astronautics, July 2016.

-16 Boffard, J. B., Lin, C. C., and Jr, C. A. D., "Application of excitation cross sections to optical plasma diagnostics," Journal of Physics D: Applied Physics, Vol. 37, No. 12, 2004, pp. R143.

-17 Kanik, I., Johnson, P. V., and James, G. K., "Electron-impact-induced emission and excitation cross sections of xenon at low energies," Journal of Physics B: Atomic, Molecular and Optical Physics, Vol. 34, No. 9, 2001, pp. 1685.

18 Trent, K. R., McDonald, M. S., Lobbia, R. B., and Gallimore, A. D., "Time-resolved Langmuir Probing of a New Lanthanum Hexaboride (LaB6) Hollow Cathode," Tech. rep., University of Michigan, Sept. 2011.

19 Stix, T. H., The Theory of Plasma Waves, 1962.

20 SensL, "An Introduction to the Silicon Photomultiplier," June 2017.

21 SensL, "J-Series High PDE and Timing Resolution, TSV Package," June 2017.

$\triangleright^{22}$ Durot, C. J., Gallimore, A. D., and Smith, T. B., "Validation and evaluation of a novel time-resolved laserinduced fluorescence technique," Review of Scientific Instruments, Vol. 85, No. 1, Jan. 2014, pp. 013508. 